\section{ON ABDOMINAL EVACUA'TION OF THE PREGNANT UTERUS BEFORE VIABILITY.}

BY VICTOR BONNEY, M.S., M.D., B.SC.LoN)., F.R.O.S., M.R.C.P.,

ASSISTANT GYNACOLOGICAL SURGEON TO THE MIDDLLSEX HOSPIFAL; SURG TOK TO THE CaELBEA HOSPITAL FOR WOMBN, HTC.

THE appreciation of the fact that an incision through the uterine wall, provided the uterine cavity is not infected, can be stanched of blood, sutured, and got to heal with perfect security and safety has immensely enlarged the scope of hysterotomy.

Practically restricted in the older days to the timorous performance of Cxsarean section, it can now be employed not only for the abdominal delivery of a living child, but for the enucleation of fibroids, single or multiple, deeply embedded in the uterine wall, for the exploratory opening up of the uterine cavity in cases of menorrhagia or metrorrhagia of obscure origin, and for the extraction of the products of gestation before viability. It is with this latter application of hysterotomy that I now deal.

\section{Conditions in which the Operation is Indioated.}

What are the circumstances under which it is preferable before viability to empty the pregnant uterus through the abdomen? In the main there are three sets of circumstances, as follows :-

1. When in addition to evaouation sterilisation of the patient is required. - In certain cases of pregnancy in tubercular women the uterus should be emptied at once and further pregnancy prevented by ligaturing or removing the tubes. As a typical case of this sort I may instance that of a woman who, having had several children, developed acute phthisis whilst carrying the last one. Treatment in a sanatorium resulted in recovery sufficient enough to allow her to return to her home and household duties, when she promptly became pregnant once more.

Again, in valvular disease of the heart with compensation, already trembling in the balance, pregnancy should be arrested as soon after its initiation as may be feasible, and further conception rendered impossible.

Much rarer than these two instances are those cases in which pregnancy is habitually followed by some dangerous disturbance of the economy, as, for instance, by nephritis, diabetes, hæmolytic anæmia, or insanity. In all of these evacuation of the uterus should be combined with a sterilising operation. Finally, cases of physical deformity incompatible with continued pregnancy are sometimes met with, such as extreme kyphosis.

The older course in all these cases was to first evacuate the uterus through the cervix or to induce abortion, and then to open the abdomen and ligature or remove the tubes. I have abandoned this practice for some years, believing it to be faulty, more especially if the pregnancy has advanced beyond the third month, and, having opened the abdomen, I now deliberately incise the uterus through its anterior wall, shell out the pregnancy, close the aterine wound with three mattress sutures and a superficial continuous suture, and then ligature and cut off the outer halves of the tubes. By this means the proceeding is shortened and simplified, and, moreover, rendered absolutely aseptic, for the uterine interior is sterile to start with, and, for the rest, I have the factors that will keep it so entirely under my command. It is doubtful to my mind whether evacuation through the cervix can ever be carried out without introducing some organisms into the uterus whilst the commonly used means of inducing abortion, the bougie and tent, almost certainly act as organismal conveyers.

2. When the pregnancy has advanoed to the fourth month or over and its termination is urgent. -The evacuation through the cervix of a pregnancy of five or six months' standing is a formidable operation. The extraction of a head of even four months' growth needs a degree of cervical dilatation which almost always splits the cervix. At five or six months sufficient dilatation cannot be obtained even by deep splitting, and the surgeon is obliged to incise the lower pole of the uterus along its anterior wall from the external os upward to the peritoneal reflection-the so-called vaginal Cæsarean section.

Vaginal Cæsarean section is an operation for the expert alone, particularly in women who have not previously borne children; but its greatest drawback is that it inflicts a wound in the uterus through a passage (the vagina) that cannot probably with certainty be rendered initially sterile, and certainly not kept sterile during an operation of come duration.

For these reasons I have for a long time now adopted the abdominal route for the removal of a pregnancy of four, five, or six months' standing in cases of gastro-hepatic or cerebro-renal toxæmia (pernicious vomiting and eclamptic nephritis) or such other gravely menacing complications of pregnancy.

3. In certain cases of pregnaney complieated by fibroide.Every now and then one comes across cases of early pregnancy complicated by fibroids in which the tumour or tumours force surgical intervention. It should be a cardinal rule in the surgery of fibroids that whenever the uterus bearing them is also pregnant the removal of the organ should be avoided if possible-it has proved its domestic and national value.

Now the uterus nearly always can be spared. Pedunculated fibroids can be removed and the pregnancy left in situ, abortion only rarely following. But this is not so if the tumour or tumours be embedded, for then their removal alone will almost certainly be followed by abortion. Moreover, the suture of the cavity or cavities left in the uterine wall after the enucleation is not likely to be satisfactory if the organ be still distended and vascular from the pregnancy within it, and further, if the organ empties itself within the next 48 hours there is a considerable chance of intraperitoneal bleeding from the blood-stanching sutures becoming loose. For this reason it has been the custom to remove the pregnant aterus with the fibroids in such cases.

But why not treat the pregnancy like another fibroid, enucleate it too, and leave the patient with her still competent organ? She can, and will, become pregnant again minus the disability of concomitant myomatous growth. Fears may be entertained that the scarred uterus will not stand the strain. They are groundless. Of all tissues I believe the uterine muscle has the most perfect healing powers. You can never find the uterine scar of a previous Cresarean section when you open the abdomen to perform the operation for a second time, and I have had two opportunities now of viewing a pregnant uterus on which I had previonsly done myomectomy, and the same was true.

\section{Conclusion.}

In conclusion, then, I feel sure that under the several circumstances I have detailed, the early pregnant uterus should be evacuated through the abdomen.

The technique is just that of a Cæsarean section, than which it is an even easier operation. The aterine incision needed is smaller, of course, the bleeding is less, and fewer mattress sutures are required to close the hole and stanch the blood-flow. I use silk (No. 4) for these sutures, and insert a continuous suture of No. 2 30-day catgut to bring into apposition the peritoneal edges: these stitches should pass deeply. One c.cm. of pituitrin should be given just before the operation and repeated during the suture of the uterus. The incision. should always be made through the anterior wall for two important reasons : first, so that the uterine wound may present towards the bladder and thus avoid adhesion to the intestine; and, secondly, so that if persistent oozing goes on from the needle punctures the uterus can be fixed to the anterior abdominal wall by two or three silk sutures along the line of the uterine incision. Oozing from the uterine incision or needle punctures is dangerous, because the effused blood attracts coils of intestine to adhere to the uterus.

Before deciding that oozing from these sites is really persistent, put the uterus back into its place and watch it for a minute or so. So long as it is outside the abdominal wound it tends to bleed, because the venous return is interfered with by the traction on the broad ligaments and the nipping of the abdominal wound edges. In this regard the uterus is like the kidney pulled through a loin wound; moreover, no uterus will retract perfectly until it is back in its proper place. 